\title{
Harvesting Multiqubit Entanglement from Ultrastrong Interactions in Circuit Quantum Electrodynamics
}

\author{
F. Armata, ${ }^{1}$ G. Calajo, ${ }^{2}$ T. Jaako, ${ }^{2}$ M. S. Kim, ${ }^{1,3}$ and P. Rabl ${ }^{2}$ \\ ${ }^{1}$ QOLS and QuEST, Blackett Laboratory, Imperial College London, London SW7 2AZ, United Kingdom \\ ${ }^{2}$ Vienna Center for Quantum Science and Technology, Atominstitut, TU Wien, 1040 Vienna, Austria \\ ${ }^{3}$ Korea Institute of Advanced Study, Dongdaemun-gu, Seoul 02455, South Korea
}

(Received 30 July 2017; published 1 November 2017)

\begin{abstract}
We analyze a multiqubit circuit QED system in the regime where the qubit-photon coupling dominates over the system's bare energy scales. Under such conditions a manifold of low-energy states with a high degree of entanglement emerges. Here we describe a time-dependent protocol for extracting these quantum correlations and converting them into well-defined multipartite entangled states of noninteracting qubits. Based on a combination of various ultrastrong-coupling effects, the protocol can be operated in a fast and robust manner, while still being consistent with experimental constraints on switching times and typical energy scales encountered in superconducting circuits. Therefore, our scheme can serve as a probe for otherwise inaccessible correlations in strongly coupled circuit QED systems. It also shows how such correlations can potentially be exploited as a resource for entanglement-based applications.
\end{abstract}

DOI: 10.1103/PhysRevLett.119.183602

Cavity QED is the study of quantum light-matter interactions with real or artificial two-level atoms coupled to a single radiation mode. In this context one is usually interested in strong interactions between excited atomic and electromagnetic states, while the trivial ground state, i.e., the vacuum state with no atomic or photonic excitations, plays no essential role. This paradigm has recently been challenged by a number of experiments [1-5], where interaction strengths comparable to the photon energy have been demonstrated. In particular, in the field of circuit QED [6,7], a single superconducting two-level system can already be coupled ultrastrongly [8-10] to a microwave resonator mode [11-17]. In this regime the physics changes drastically and even in the ground state various nontrivial effects like spontaneous vacuum polarization [18-20], light-matter decoupling [21,22], and different degrees of entanglement [22-25] can occur. However, compared to the vast literature on cavity QED systems in the weakly coupled regime, the opposite limit of extremely strong interactions is to a large extent still unexplored. As a consequence, ideas for how ultrastrong coupling (USC) effects can be controlled and exploited for practical applications are limited [26-31].

In this Letter we consider a prototype circuit QED system consisting of multiple flux qubits coupled to a single mode of a microwave resonator. It has recently been shown that in the USC regime this circuit exhibits a manifold of

Published by the American Physical Society under the terms of the Creative Commons Attribution 4.0 International license. Further distribution of this work must maintain attribution to the author(s) and the published article's title, journal citation, and DOI. nonsuperradiant ground and low-energy states with a high degree of multiqubit entanglement [22]. This entanglement, however, is a priori not of any particular use, since any attempt to locally manipulate or measure the individual qubits would necessarily introduce a severe perturbation to the strongly coupled system. For this reason we describe the implementation of an entanglement-harvesting protocol [32-38], which extracts quantum correlations from USC states and converts these correlations into equivalent multipartite entangled states of decoupled qubits. The protocol combines adiabatic and nonadiabatic parameter variations and exploits the counterintuitive decoupling of qubits and photons at very strong interactions [22] to make the entanglement extraction scheme intrinsically robust and consistent with experimentally available tuning capabilities. The extracted Dicke and singlet states belong to a family of robust multipartite entangled states $[39,40]$ and form, for example, a resource for Heisenberg-limited metrology applications [41]. More generally, our analysis shows, how the interplay between different USC effects can contribute to the realization of nontrivial control tasks in a strongly interacting cavity QED system.

Model.-We consider a circuit QED system as shown in Fig. 1(a), where a single mode $L C$ resonator with capacitance $C$ and inductance $L$ is coupled collectively to an even number of $N=2,4,6, \ldots$ flux qubits. This circuit is described by the Hamiltonian $[42,43]$

$$
\mathcal{H}=\frac{Q_{r}^{2}}{2 C}+\frac{\left(\Phi_{r}-\Phi_{0} \sum_{i=1}^{N} \varphi_{i}\right)^{2}}{2 L}+\sum_{i=1}^{N} H_{q}^{(i)},
$$

where $Q_{r}$ and $\Phi_{r}$ are charge and generalized flux operators for the resonator obeying $\left[\Phi_{r}, Q_{r}\right]=i \hbar$, and $\Phi_{0}=\hbar /(2 e)$ 

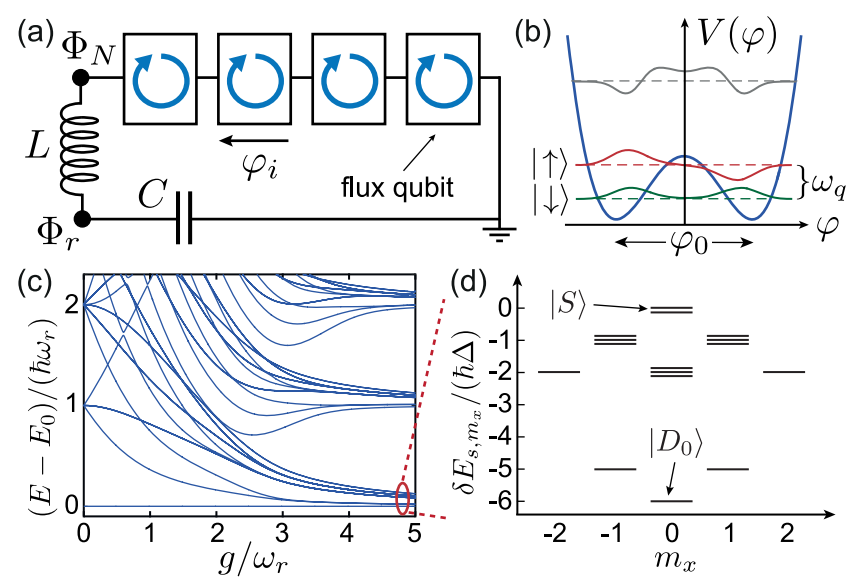

FIG. 1. (a) Sketch of the multiqubit circuit QED setup considered in this Letter. (b) Each flux qubit is represented by the two lowest states $|\downarrow\rangle$ and $|\uparrow\rangle$ of an effective double-well potential for the phase variable $\varphi$. Under this two-level approximation the inductive coupling $\left(\Phi_{r}-\Phi_{N}\right)^{2} /(2 L)$, where $\Phi_{N}=\Phi_{0} \sum_{i=1}^{N} \varphi_{i}$, gives rise to the cavity QED Hamiltonian (2). (c) Energy spectrum (with respect to the ground-state energy $E_{0}$ ) of the extended Dicke model (3) as a function of the coupling strength $g$ for $N=4$ and $\omega_{q}=\omega_{r}$. (d) Ordering of the lowest energy states in the USC regime as determined by Eq. (4) for the case $N=4$. The multiple lines indicate the two- and threefold degeneracy of states with total angular momentum $s=0$ and $s=1$, respectively.

is the reduced flux quantum. For each qubit, $H_{q}^{(i)}$ denotes the free Hamiltonian and $\varphi_{i}$ is the difference of the superconducting phase across the qubit's subcircuit. As usual we assume that the qubit dynamics can be restricted to the two lowest tunneling states $|\downarrow\rangle$ and $|\uparrow\rangle$ of a symmetric double-well potential [cf. Fig. 1(b)]. Under this approximation and writing $\Phi_{r}=\sqrt{\hbar /\left(2 C \omega_{r}\right)}\left(a+a^{\dagger}\right)$ and $Q_{r}=i \sqrt{\hbar C \omega_{r} / 2}\left(a^{\dagger}-a\right)$, where $\omega_{r}=\sqrt{1 / L C}$ is the resonator frequency and $a$ and $a^{\dagger}$ are the annihilation and creation operators, we obtain

$$
\begin{aligned}
\mathcal{H}= & \hbar \omega_{r} a^{\dagger} a+\hbar \sum_{i=1}^{N} \frac{g_{i}}{2}\left(a^{\dagger}+a\right) \sigma_{x}^{i} \\
& +\hbar \sum_{i=1}^{N} \frac{\omega_{q}^{i}}{2} \sigma_{z}^{i}+\hbar \sum_{i, j=1}^{N} \frac{g_{i} g_{j}}{4 \omega_{r}} \sigma_{x}^{i} \sigma_{x}^{j} .
\end{aligned}
$$

Here $\sigma_{k}^{i}$ are Pauli operators and $\omega_{q}^{i}$ are the qubit-level splittings. The second term in Eq. (2) accounts for the collective qubit-resonator interaction with couplings $g_{i}=\Phi_{0} \sqrt{\left|\varphi_{0}^{i}\right|^{2} \omega_{r} /(2 \hbar L)}$, where $\varphi_{0}^{i}=2\left\langle\downarrow_{i}\left|\varphi_{i}\right| \uparrow_{i}\right\rangle$. The condition $g_{i}>\omega_{r}, \omega_{q}^{i}$ can be reached with an appropriate flux-qubit design $[14,15,18,27,46,47]$, and the $g_{i}(t)$ and $\omega_{q}^{i}(t)$ can be individually tuned by controlling the matrix element $\varphi_{0}^{i}$ and the height of the tunnel barrier via local magnetic fluxes [27,36]. A specific four-junction qubit design $[47,48]$, which combines strong interactions with a high degree of tunability, is detailed in the Supplemental Material [43]. Finally, the last contribution in Eq. (2) represents an additional qubit-qubit interaction, which is usually neglected for cavity QED systems with weak or moderately strong couplings. However, this term is crucial in the USC regime and it is responsible for the nontrivial ground-state correlations that are at the focus of the present Letter.

USC spectrum.-We are primarily interested in a symmetric configuration, i.e., $g_{i}=g$ and $\omega_{q}^{i}=\omega_{q}$. In this case the Hamiltonian (2) can be expressed in terms of collective angular momentum operators $S_{k}=\sum_{i} \sigma_{k}^{i} / 2$ and reduces to the extended Dicke Hamiltonian [22]

$$
\mathcal{H}=\hbar \omega_{r} a^{\dagger} a+\hbar g\left(a^{\dagger}+a\right) S_{x}+\hbar \omega_{q} S_{z}+\hbar \frac{g^{2}}{\omega_{r}} S_{x}^{2} .
$$

For $g \ll \omega_{r}, \omega_{q}$ we can make a rotating wave approximation and obtain the standard Tavis-Cummings model of cavity QED with a trivial ground state $|G\rangle=|n=0\rangle \otimes$ $|\downarrow\rangle^{\otimes N}$. If in addition $\left|\omega_{q}-\omega_{r}\right| \gg g$, all excited states are also essentially decoupled and the qubits can be individually prepared, manipulated, and measured by additional control fields. In the opposite limit, $g \gg \omega_{r}, \omega_{q}$, the coupling terms $\sim S_{x}$ and $\sim S_{x}^{2}$ dominate and the level structure changes completely. This is illustrated in Fig. 1(c), which shows that for couplings $g / \omega_{r} \gtrsim 3$ the spectrum separates into manifolds of $2^{N}$ nearly degenerate states. The eigenstates in this regime are displaced photon number states, $\left|\Psi_{s, m_{x}, n}\right\rangle \simeq e^{-g / \omega_{r}\left(a^{\dagger}-a\right) S_{x}}|n\rangle \otimes\left|s, m_{x}\right\rangle$, with energies $E_{s, m_{x}, n} \simeq \hbar \omega_{r} n+\delta E_{s, m_{x}}^{(n)}$ [22]. Here $s$ is the total spin and $m_{x}=-s, \ldots, s$ the spin projection quantum number; i.e., $S_{x}\left|s, m_{x}\right\rangle=m_{x}\left|s, m_{x}\right\rangle$. Within the lowest manifold, the remaining level splittings are given by

$$
\delta E_{s, m_{x}}^{(0)}=\hbar \Delta\left[m_{x}^{2}-s(s+1)\right], \quad \Delta=\frac{\omega_{q}^{2} \omega_{r}}{2 g^{2}},
$$

and the resulting ordering of the states is shown in Fig. 1(d) for $N=4$ qubits. Thus, for even qubit numbers $N$, the ground state in the USC regime is of the form $|\tilde{G}\rangle \simeq|n=0\rangle \otimes\left|D_{0}\right\rangle$, where $\left|D_{0}\right\rangle=\left|s=N / 2, m_{x}=0\right\rangle$ denotes the fully symmetric Dicke state with vanishing projection along $x$. Importantly, this state exhibits a high degree of qubit-qubit entanglement, while it remains almost completely decoupled from the cavity field [22]. Our goal is now to identify a suitable protocol for converting this state into an equivalent state of the decoupled system, where it becomes available as an entanglement resource for further use.

Entanglement harvesting.-Figure 2(a) shows a general pulse sequence for implementing the entanglementharvesting protocol through variations of $\omega_{q}(t)$ and $g(t)$. For this protocol the system is initialized in the ground state $|G\rangle$ of the weakly coupled system, where the qubits are far 

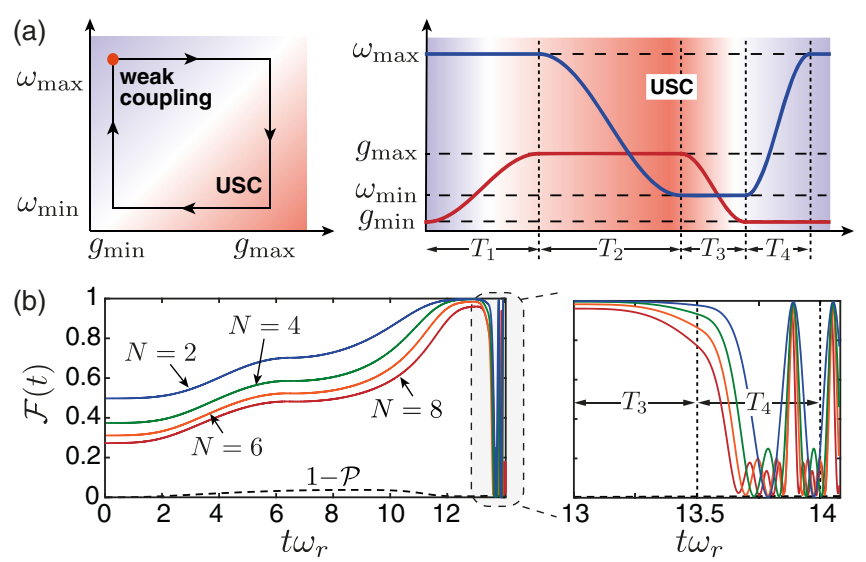

FIG. 2. (a) General pulse sequence for the qubit parameters $\omega_{q}(t)$ and $g(t)$ considered for the implementation of the entanglement harvesting protocol. (b) The fidelity $\mathcal{F}(t)$ is plotted as a function of time and for different qubit numbers. The dashed line indicates the quantity $1-\mathcal{P}(t)$, where $\mathcal{P}(t)=\operatorname{Tr}\left\{\rho_{q}^{2}(t)\right\}$ is the purity of the reduced qubit state $\rho_{q}(t)=\operatorname{Tr}_{r}\{\rho(t)\}$ for the case $N=4$. It shows that after an intermediate stage of finite qubitresonator entanglement, the purity of the qubit state is almost fully restored when the system enters deep into the USC regime. For all values of $N$ the same parameters $\omega_{\max } / \omega_{r}=20, \omega_{\min } / \omega_{r}=0.5$, $g_{\max } / \omega_{r}=4.5, g_{\min } / \omega_{r}=0.1$ and times intervals $T_{1}=T_{2}=$ $6.5 \omega_{r}^{-1}$ and $T_{3}=T_{4}=0.5 \omega_{r}^{-1}$ have been assumed.

detuned from the cavity, $\omega_{q}=\omega_{\max } \gg \omega_{r}$, and the coupling is set to a minimal value, $g=g_{\min }<\omega_{r}$. In the first two steps, $T_{1}$ and $T_{2}$, the system is adiabatically tuned into the USC regime with a maximal coupling $g_{\max }>\omega_{r}$ and a low value of the qubit frequency $\omega_{\min } \lesssim \omega_{r}$. This process prepares the system in the USC ground state $|\tilde{G}\rangle$. In the successive steps, $T_{3}$ and $T_{4}$, the qubits and the resonator mode are separated again, but now in the reverse order and using nonadiabatic parameter variations. Ideally, during this part of the protocol the system simply remains in state $|\tilde{G}\rangle$ and becomes the desired excited state of the weakly coupled system at the final time $T_{f}=\sum_{n=1}^{4} T_{n}$. This general sequence achieves two main goals. First, the adiabatic preparation stage can be implemented very rapidly, since it must only be slow compared to the fast time scales set by $\omega_{\max }^{-1}$ and $g_{\max }^{-1}$. At the same time the nonadiabatic decoupling processes only need to be fast compared to the slow time scales $\omega_{r}^{-1}, \omega_{\min }^{-1}$, and $g_{\min }^{-1}$. This second condition is most crucial for a time-dependent control of USC systems, since it makes the required switching times experimentally accessible and consistent with the two-level approximation assumed in our theoretical model.

In Fig. 2(b) we plot the fidelity $\mathcal{F}(t)=\operatorname{Tr}\left\{\rho(t)\left|D_{0}\right\rangle\left\langle D_{0}\right|\right\}$, where $\rho(t)$ is the density operator of the full system, for a specific set of pulse parameters listed in the figure caption. We see that the entanglement extraction fidelity (EEF) $\mathcal{F}_{E}=\max \left\{\mathcal{F}(t) \mid t \geq T_{f}\right\}$, i.e., the maximal fidelity after the decoupling step, reaches near perfect values of $\mathcal{F}_{E} \simeq$ 0.95-0.99 for different numbers of qubits, without any further fine-tuning of the control pulses. Note that the fidelity oscillations at the end of the sequence are simply due to the fact that $\left|D_{0}\right\rangle$ is not an eigenstate of the bare qubit Hamiltonian, $H_{q}=\omega_{q} S_{z}$. However, this evolution does not affect the purity or the degree of entanglement of the final qubit state and can be undone by local qubit rotations.

Experimental considerations.-For a possible experimental implementation of the protocol we consider qubits with a frequency of $\omega_{\max } /(2 \pi) \approx 10 \mathrm{GHz}$ coupled to a lumped-element resonator of frequency $\omega_{r} /(2 \pi)=$ $500 \mathrm{MHz}$. The required maximal coupling strength of $g_{\max } \approx 4.5 \omega_{r} \approx 2 \pi \times 2.25 \mathrm{GHz}$ is then consistent with experimentally demonstrated values $[14,15]$. For these parameters, the nonadiabatic switching times assumed in Fig. 2(b) correspond to $T_{3,4} \simeq 0.16 \mathrm{~ns}$. These switching times are within reach of state-of-the-art waveform generators and a sinusoidal modulation of flux qubits on such time scales has already been demonstrated [49]. At the same time the duration of the whole protocol, $T_{f}=$ $15 / \omega_{r} \approx 5 \mathrm{~ns}$, is still much faster than typical flux qubit coherence times of 1-100 $\mu \mathrm{s}$ [50] or the lifetime of a photon, $T_{\mathrm{ph}}=Q / \omega_{r}$, in a microwave resonator of quality factor $Q=10^{4}-10^{6}$. Therefore, although many experimental techniques for implementing and operating circuit QED systems in the USC regime are still under development, these estimates clearly demonstrate the feasibility of realizing high-fidelity control operations in such devices.

In practice additional limitations might arise from the lack of complete tunability of $g(t)$ and $\omega_{q}(t)$. This is illustrated in Fig. 3(a), which shows the evolution of the lowest eigenenergies during different stages of the protocol for the case $N=2$ and a nonvanishing value of $g_{\min }$. In this case the appearance of several avoided crossings during the final ramp-up step prevents a fully nonadiabatic decoupling. In Fig. 3(b) we plot the resulting EEF for varying $g_{\min }$ and $T_{4}$. This plot demonstrates the expected trade-off between the residual coupling and the minimal switching time, but also that the protocol is rather robust and fidelities of $\mathcal{F}_{E} \sim 0.9$ are still possible for minimal couplings of a few hundred $\mathrm{MHz}$ or switching times approaching $~ 1 \mathrm{~ns}$. Similar conclusions are obtained when a partial dependence between the pulses for $g(t)$ and $\omega_{q}(t)$ or nonuniform couplings $g_{i}(t)$ and frequencies $\omega_{q}^{i}(t)$ due to fabrication uncertainties are taken into account. Numerical simulations of the protocol under such realistic experimental conditions [43] demonstrate that no precise fine-tuning of the system parameters is required.

Extracting entanglement from a thermal state.-The above-considered protocol relies on a rather low resonator frequency $\omega_{r}$ in order to enhance both $g / \omega_{r}$ as well as the nonadiabatic switching times. This implies that even at temperatures of $T=20 \mathrm{mK}$ the equilibrium populations of higher resonator states with $n \geq 1$ cannot be neglected. 
(a)
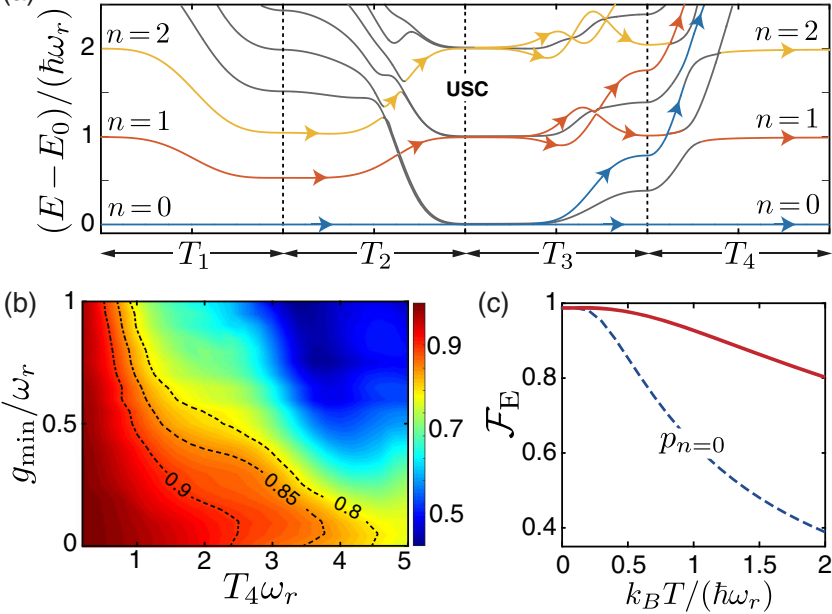

FIG. 3. (a) Evolution of the lowest eigenvalues during different stages of the protocol for the case $N=2$. Here $g_{\min } / \omega_{r}=0.2$, $\omega_{\min } / \omega_{r}=0.4$, and in the final step of the protocol $\omega_{\max } / \omega_{r}=5$. For clarity only the $s=1$ states are shown and all time intervals have been stretched to equal lengths. For different initial photon number states $|n\rangle$, the colored segments and arrows indicate the ideal evolution of the systems, which maximizes the probability to end up in the qubit state $\left|D_{0}\right\rangle=(|\uparrow \uparrow\rangle-|\downarrow \downarrow\rangle) / \sqrt{2}$. Nonadiabatic crossings occur during the fast decoupling steps $\left(T_{3}\right.$ and $\left.T_{4}\right)$, but also for small avoided crossings in the excited state manifolds during the preparation step $\left(T_{2}\right)$. (b) Plot of the EEF for varying $T_{4}\left(=T_{3}\right)$ and $g_{\min }$ and for $N=4$. (c) EEF (solid line) for a resonator mode, which is initially in a thermal state at temperature $T$, for $N=4$. The dashed line indicates the corresponding population of the ground state manifold. All the other pulse parameters in panels (a), (b), and (c) are the same as in Fig. 2(b).

In Fig. 3(c) we plot the EEF as a function of the temperature $T$, assuming an initial resonator state $\rho_{\text {th }}=\sum_{n} p_{n}|n\rangle\langle n|$, where $p_{n}=\bar{n}^{n} /(1+\bar{n})^{n+1}$ is the thermal distribution for a mean excitation number $\bar{n}=1 /\left(e^{\hbar \omega_{r} / k_{B} T}-1\right)$. We see that the EEF is significantly higher than one would naively expect from the initial population in the ground state $|G\rangle$. The origin of this surprising effect can be understood from the eigenvalue plot in Fig. 3(a). For example, the weakcoupling eigenstate $|n=1\rangle \otimes|\downarrow\rangle \otimes N$ is efficiently mapped on the corresponding USC state $|n=1\rangle \otimes \mid s=N / 2$, $\left.m_{x}=0\right\rangle$, passing only through a weak, higher-order avoided crossing. Therefore, the intermediate-and as a result also the final-qubit state is one with the resonator being in state $|1\rangle$. Although for higher photon numbers the avoided crossings become more relevant, the protocol still approximately implements the mapping $|n\rangle \otimes|\downarrow\rangle^{\otimes N} \rightarrow$ $|n\rangle \otimes\left|s=N / 2, m_{x}=0\right\rangle$, independent of the resonator state $|n\rangle$. This feature makes it rather insensitive to thermal occupations and avoids additional active cooling methods for initializing the system in state $|G\rangle$.

Entanglement protection.-Figure 1(d) shows that apart from the ground state $|\tilde{G}\rangle$ there are many other highly entangled states within the lowest USC manifold.
Of particular interest is the energetically highest state $|\tilde{E}\rangle=|n=0\rangle \otimes|S\rangle$, where $|S\rangle$ is a singlet state with total angular momentum $s=0$ and $S_{z}|S\rangle=S_{x}|S\rangle=0$. Therefore, once prepared, this state is an exact dark state of Hamiltonian (3) and remains decoupled from the cavity field in all parameter regimes. Although this state is not connected to any of the bare qubit states in a simple adiabatic way, it can still be harvested by an adopted protocol, as described in Fig. 4(a) for the case $N=4$. For this protocol the system is initially prepared in the excited state $\left|\Psi_{0}\right\rangle=|0\rangle \otimes|\uparrow \uparrow \downarrow \downarrow\rangle$ and in a first step the qubit states are lowered below the resonator frequency in order to avoid further level crossings with higher-photon-number states. The increase of the coupling combined with a frequency offset to break the angular momentum conservation then evolves the system into a state with $s=0$ already for moderate couplings of $g / \omega_{r} \approx 1.8$. Note that for $N \geq 4$ there are multiple degenerate USC states with $s=0$ [51,52] [cf. Fig. 1(d)], out of which the protocol selects a specific superposition [43].

Although the harvesting protocol for state $|S\rangle$ loses some of the robustness of the ground-state protocol, it adds an
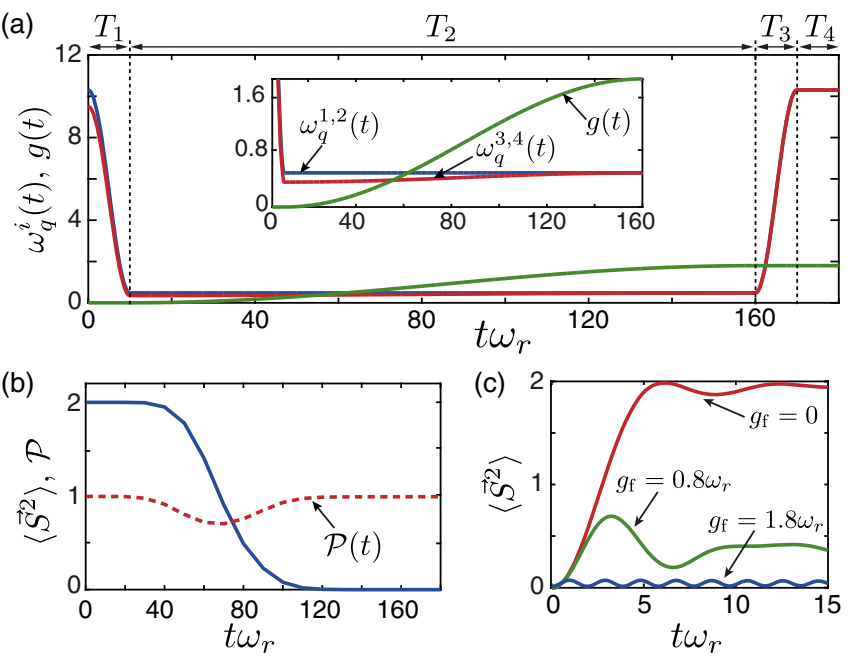

FIG. 4. (a) Pulse sequence for harvesting the 4-qubit entangled state $|S\rangle$ with total angular momentum $s=0$. As shown in the inset, during the first part of the protocol a finite difference between the qubit frequencies $\omega_{q}^{1,2}$ and $\omega_{q}^{3,4}$ is used to break the symmetry and couple different angular momentum states. (b) The expectation value of the total spin $\left\langle\vec{S}^{2}(t)\right\rangle$ (solid line) and the purity of the reduced qubit state $\mathcal{P}(t)$ (dashed line) are plotted for the pulse sequence shown in (a) and for an initial state $\left|\Psi_{0}\right\rangle=|0\rangle \otimes|\uparrow \uparrow \downarrow \downarrow\rangle$. (c) Evolution of the extracted state $|0\rangle \otimes$ $|S\rangle$ (characterized by the expectation value of the total spin) after the protocol for different final values of the couplings $g_{f}$. For this plot an average over random distributions of the qubit frequencies, $\omega_{q}^{i}=\omega_{q}\left(1+\epsilon_{i}\right)$, has been assumed, where $\omega_{q} / \omega_{r}=10$ and the $\epsilon_{i}$ are chosen randomly from the interval $[-0.05,0.05]$. For very strong couplings, the residual oscillations indicate that all transitions induced by the nonuniform $\epsilon_{i}$ from state $|S\rangle$ to other states are highly detuned. 
important feature. By retaining a finite coupling $g_{f}=$ $g\left(t=T_{f}\right) \sim \omega_{r}$ at the end of the protocol, the extracted dark state $|S\rangle$ is energetically separated from all other states with $s \neq 0$ and it is thereby protected against small frequency fluctuations. This effect is illustrated in Fig. 4(c), which shows the evolution of the extracted state $|S\rangle$ in the presence of small random shifts of the individual qubit frequencies. For $g_{f}=0$ this leads to dephasing of the qubits and a rapid transition out of the $s=0$ subspace. This dephasing can be substantially suppressed by keeping the coupling at a finite value. Thus, this example shows that USC effects can be used not only to generate complex multiqubit entangled states, but also to protect them.

Conclusion.-We have presented a protocol for extracting well-defined multiqubit-entangled states from the ground-state manifold of an ultrastrongly coupled circuit QED system. The detailed analysis of this protocol illustrates, how various-so far unexplored-USC effects can contribute to a robust generation and protection of complex multiqubit states. These principles can serve as a guideline for many other preparation, storage, and control operations in upcoming USC circuit QED experiments with two or more qubits.

This work was supported by the Austrian Science Fund (FWF) through the SFB FoQuS, Grant No. F40, the DK CoQuS, Grant No. W 1210, and the START Grant No. Y 591-N16, and the People Programme (Marie Curie Actions) of the European Union's Seventh Framework Programme (FP7/2007-2013) under REA Grant No. 317232. F. A. wishes to express his gratitude to the Quantum Optics Theory Group of Atominstitut (TU Wien) for the warm hospitality received during his numerous visits. M. S. K. acknowledges support from the UK EPSRC Grant No. EP/ K034480/1 and the Royal Society.

[1] Y. Todorov, A. M. Andrews, R. Colombelli, S. De Liberato, C. Ciuti, P. Klang, G. Strasser, and C. Sirtori, Ultrastrong Light-Matter Coupling Regime with Polariton Dots, Phys. Rev. Lett. 105, 196402 (2010).

[2] T. Schwartz, J. A. Hutchison, C. Genet, and T. W. Ebbesen, Reversible Switching of Ultrastrong Light-Molecule Coupling, Phys. Rev. Lett. 106, 196405 (2011).

[3] M. Geiser, F. Castellano, G. Scalari, M. Beck, L. Nevou, and J. Faist, Ultrastrong Coupling Regime and Plasmon Polaritons in Parabolic Semiconductor Quantum Wells, Phys. Rev. Lett. 108, 106402 (2012).

[4] G. Scalari, C. Maissen, S. Cibella, R. Leoni, C. Reichl, W. Wegscheider, M. Beck, and J. Faist, THz ultrastrong lightmatter coupling, Il Nuovo Saggiatore 31, 4 (2015).

[5] Q. Zhang, M. Lou, X. Li, J. L. Reno, W. Pan, J. D. Watson, M. J. Manfra, and J. Kono, Collective non-perturbative coupling of 2D electrons with high-quality-factor terahertz cavity photons, Nat. Phys. 12, 1005 (2016).

[6] A. Wallraff, D. I. Schuster, A. Blais, L. Frunzio, R. S. Huang, J. Majer, S. Kumar, S. M. Girvin, and R. J. Schoelkopf, Strong coupling of a single photon to a superconducting qubit using circuit quantum electrodynamics, Nature (London) 431, 162 (2004).

[7] A. Blais, R.-S. Huang, A. Wallraff, S. M. Girvin, and R. J. Schoelkopf, Cavity quantum electrodynamics for superconducting electrical circuits: An architecture for quantum computation, Phys. Rev. A 69, 062320 (2004).

[8] C. Ciuti, G. Bastard, and I. Carusotto, Quantum vacuum properties of the intersubband cavity polariton field, Phys. Rev. B 72, 115303 (2005).

[9] J. Casanova, G. Romero, I. Lizuain, J. J. García-Ripoll, and E. Solano, Deep Strong Coupling Regime of the Jaynes-Cummings Model, Phys. Rev. Lett. 105, 263603 (2010).

[10] Note that in this Letter we do not further distinguish between the USC [8] and the deep strong-coupling regime [9].

[11] T. Niemczyk et al., Circuit quantum electrodynamics in the ultrastrong-coupling regime, Nat. Phys. 6, 772 (2010).

[12] P. Forn-Diaz, J. Lisenfeld, D. Marcos, J. J. Garcia-Ripoll, E. Solano, C. J. P. M. Harmans, and J. E. Mooij, Observation of the Bloch-Siegert Shift in a Qubit-Oscillator System in the Ultrastrong Coupling Regime, Phys. Rev. Lett. 105, 237001 (2010).

[13] A. Baust, E. Hoffmann, M. Haeberlein, M. J. Schwarz, P. Eder, J. Goetz, F. Wulschner, E. Xie, L. Zhong, F. Quijandría, D. Zueco, J.-J. García Ripoll, L. García-Álvarez, G. Romero, E. Solano, K. G. Fedorov, E. P. Menzel, F. Deppe, A. Marx, and R. Gross, Ultrastrong coupling in tworesonator circuit QED, Phys. Rev. B 93, 214501 (2016).

[14] P. Forn-Diaz, J. J. García-Ripoll, B. Peropadre, M. A. Yurtalan, J.-L. Orgiazzi, R. Belyansky, C. M. Wilson, and A. Lupascu, Ultrastrong coupling of a single artificial atom to an electromagnetic continuum, Nat. Phys. 13, 39 (2017).

[15] F. Yoshihara, T. Fuse, S. Ashhab, K. Kakuyanagi, S. Saito, and K. Semba, Superconducting qubit-oscillator circuit beyond the ultrastrong-coupling regime, Nat. Phys. 13, 44 (2017).

[16] Z. Chen, Y. Wang, T. Li, L. Tian, Y. Qiu, K. Inomata, F. Yoshihara, S. Han, F. Nori, J. S. Tsai, and J. Q. You, Multiphoton sideband transitions in an ultrastrongly coupled circuit quantum electrodynamics system, Phys. Rev. A 96, 012325 (2017).

[17] S. J. Bosman, M. F. Gely, V. Singh, A. Bruno, D. Bothner, and G. A. Steele, Multi-mode ultra-strong coupling in circuit quantum electrodynamics, arXiv:1704.06208.

[18] P. Nataf and C. Ciuti, Vacuum Degeneracy of a Circuit QED System in the Ultrastrong Coupling Regime, Phys. Rev. Lett. 104, 023601 (2010).

[19] P. Nataf and C. Ciuti, No-go theorem for superradiant quantum phase transitions in cavity QED and counterexample in circuit QED, Nat. Commun. 1, 72 (2010).

[20] M. Bamba, K. Inomata, and Y. Nakamura, Superradiant Phase Transition in a Superconducting Circuit in Thermal Equilibrium, Phys. Rev. Lett. 117, 173601 (2016).

[21] S. De Liberato, Light-Matter Decoupling in the Deep Strong Coupling Regime: The Breakdown of the Purcell Effect, Phys. Rev. Lett. 112, 016401 (2014).

[22] T. Jaako, Z.-L. Xiang, J. J. Garcia-Ripoll, and P. Rabl, Ultrastrong coupling phenomena beyond the Dicke model, Phys. Rev. A 94, 033850 (2016). 
[23] G. Levine and V. N. Muthukumar, Entanglement of a qubit with a single oscillator mode, Phys. Rev. B 69, 113203 (2004).

[24] A. P. Hines, C. M. Dawson, R. H. McKenzie, and G. J. Milburn, Entanglement and bifurcations in Jahn-Teller models, Phys. Rev. A 70, 022303 (2004).

[25] S. Ashhab and F. Nori, Qubit-oscillator systems in the ultrastrong-coupling regime and their potential for preparing nonclassical states, Phys. Rev. A 81, 042311 (2010).

[26] P. Nataf and C. Ciuti, Protected Quantum Computation with Multiple Resonators in Ultrastrong Coupling Circuit QED, Phys. Rev. Lett. 107, 190402 (2011).

[27] G. Romero, D. Ballester, Y. M. Wang, V. Scarani, and E. Solano, Ultrafast Quantum Gates in Circuit QED, Phys. Rev. Lett. 108, 120501 (2012).

[28] T. H. Kyaw, S. Felicetti, G. Romero, E. Solano, and L.-C. Kwek, Scalable quantum memory in the ultrastrong coupling regime, Sci. Rep. 5, 8621 (2015).

[29] Y. Wang, J. Zhang, C. Wu, J. Q. You, and G. Romero, Holonomic quantum computation in the ultrastrong-coupling regime of circuit QED, Phys. Rev. A 94, 012328 (2016).

[30] Y. Wang, C. Guo, G.-Q. Zhang, G. Wang, and C. Wu, Ultrafast quantum computation in ultrastrongly coupled circuit QED systems, Sci. Rep. 7, 44251 (2017).

[31] R. Stassi and F. Nori, Quantum memory in the ultrastrongcoupling regime via parity symmetry breaking, arXiv: 1703.08951

[32] B. Reznik, A. Retzker, and J. Silman, Violating Bell's inequalities in vacuum, Phys. Rev. A 71, 042104 (2005).

[33] M. Han, S. J. Olson, and J. P. Dowling, Generating entangled photons from the vacuum by accelerated measurements: Quantum-information theory and the Unruh-Davies effect, Phys. Rev. A 78, 022302 (2008).

[34] A. Auer and G. Burkard, Entangled photons from the polariton vacuum in a switchable optical cavity, Phys. Rev. B 85, 235140 (2012).

[35] S. J. Olson and T. C. Ralph, Extraction of timelike entanglement from the quantum vacuum, Phys. Rev. A 85, 012306 (2012).

[36] C. Sabin, B. Peropadre, M. del Rey, and E. Martin-Martinez, Extracting Past-Future Vacuum Correlations Using Circuit QED, Phys. Rev. Lett. 109, 033602 (2012).

[37] G. Salton, R. B. Mann, and N. C. Menicucci, Accelerationassisted entanglement harvesting and rangefinding, New J. Phys. 17, 035001 (2015).

[38] L. Dai, W. Kuo, and M. C. Chung, Extracting entangled qubits from Majorana fermions in quantum dot chains through the measurement of parity, Sci. Rep. 5, 11188 (2015).
[39] O. Gühne, F. Bodoky, and M. Blaauboer, Multiparticle entanglement under the influence of decoherence, Phys. Rev. A 78, 060301(R) (2008).

[40] M. Bergmann and O. Gühne, Entanglement criteria for Dicke states, J. Phys. A 46, 385304 (2013).

[41] G. Toth and I. Apellaniz, Quantum metrology from a quantum information science perspective, J. Phys. A 47, 424006 (2014).

[42] U. Vool and M. Devoret, Introduction to quantum electromagnetic circuits, Int. J. Circuit Theory Appl. 45, 897 (2017).

[43] See Supplemental Material at http://link.aps.org/ supplemental/10.1103/PhysRevLett.119.183602 for additional details about the circuit design and the performance of the protocol under nonideal conditions, which includes Refs. $[44,45]$.

[44] F. Beaudoin, J. M. Gambetta, and A. Blais, Dissipation and ultrastrong coupling in circuit QED, Phys. Rev. A 84, 043832 (2011).

[45] A. Ridolfo, M. Leib, S. Savasta, and M. J. Hartmann, Photon Blockade in the Ultrastrong Coupling Regime, Phys. Rev. Lett. 109, 193602 (2012).

[46] J. Bourassa, J. M. Gambetta, A. A. Abdumalikov, O. Astafiev, Y. Nakamura, and A. Blais, Ultrastrong coupling regime of cavity QED with phase-biased flux qubits, Phys. Rev. A 80, 032109 (2009).

[47] B. Peropadre, D. Zueco, D. Porras, and J. J. Garcia-Ripoll, Nonequilibrium and Nonperturbative Dynamics of Ultrastrong Coupling in Open Lines, Phys. Rev. Lett. 111, 243602 (2013).

[48] Y. Qiu, W. Xiong, X.-L. He, T.-F. Li, and J. Q. You, Four-junction superconducting circuit, Sci. Rep. 6, 28622 (2016).

[49] C. M. Wilson, G. Johansson, A. Pourkabirian, J. R. Johansson, T. Duty, F. Nori, and P. Delsing, Observation of the dynamical Casimir effect in a superconducting circuit, Nature (London) 479, 376 (2011).

[50] F. Yan, S. Gustavsson, A. Kamal, J. Birenbaum, A. P. Sears, D. Hover, D. Rosenberg, G. Samach, T. J. Gudmundsen, J. L. Yoder, T. P. Orlando, J. Clarke, A. J. Kerman, and W. D. Oliver, The flux qubit revisited to enhance coherence and reproducibility, Nat. Commun. 7, 12964 (2016).

[51] R. H. Dicke, Coherence in spontaneous radiation processes, Phys. Rev. 93, 99 (1954).

[52] F. T. Arecchi, E. Courtens, R. Gilmore, and H. Thomas, Atomic coherent states in quantum optics, Phys. Rev. A 6, 2211 (1972). 